MATHEMATICS OF COMPUTATION

Volume 79, Number 270, April 2010, Pages 845-855

S 0025-5718(09)02299-6

Article electronically published on September 18, 2009

\title{
POSITIVE INTERPOLATORY QUADRATURE RULES GENERATED BY SOME BIORTHOGONAL POLYNOMIALS
}

\author{
D. S. LUBINSKY AND A. SIDI
}

\begin{abstract}
Interpolatory quadrature rules whose abscissas are zeros of a biorthogonal polynomial have proved to be useful, especially in numerical integration of singular integrands. However, the positivity of their weights has remained an open question, in some cases, since 1980. We present a general criterion for this positivity. As a consequence, we establish positivity of the weights in a quadrature rule introduced by the second author in 1980, generated by a polynomial that is biorthogonal to $(\log x)^{j}, 0 \leq j \leq n-1$.
\end{abstract}

\section{INTRODUCTION AND RESULTS}

Let $(a, b)$ be a real interval and $w:(a, b) \rightarrow(0, \infty)$ be such that $\int_{a}^{b} x^{j} w(x) d x$ is defined and finite for $0 \leq j \leq n$. Let $p_{n}$ be a monic polynomial with distinct zeros $\left\{x_{j}\right\}_{j=1}^{n}$ in $(a, b)$. Then, as an approximation to $\int_{a}^{b} f(x) w(x) d x$, we may determine an interpolatory quadrature rule

$$
I_{n}[f]=\sum_{j=1}^{n} \lambda_{j} f\left(x_{j}\right)
$$

that integrates exactly polynomials $P$ of degree $\leq n-1$ :

$$
I_{n}[P]=\int_{a}^{b} P w .
$$

We say that $I_{n}$ is the interpolatory rule generated by $p_{n}$ and $w$. Of course the classical example is Gauss quadrature, where $p_{n}$ is the $n$th orthogonal polynomial for the weight $w$, but there are many other useful such quadratures.

When $p_{n}$ is a biorthogonal polynomial, that is, when

$$
\int_{a}^{b} p_{n} \varphi_{j} w=0, \quad 1 \leq j \leq n
$$

for some functions $\left\{\varphi_{j}\right\}_{j=1}^{n}$, the numerical efficacy of such rules has been demonstrated by Sidi [12, [13], and by Sidi and Lubinsky [17. We note that in these papers, the $p_{n}$ are derived by applying the transformations of Levin and of Sidi to appropriate moment series of Stieltjes functions. For a brief summary of these transformations, see for example [15, Chapter 19].

Received by the editor October 7, 2008.

2000 Mathematics Subject Classification. Primary 41A55, 65D30, 65B99, 42C99.

Key words and phrases. Biorthogonal polynomials, interpolatory quadrature rules, positive weights.

Research supported by NSF grant DMS0400446 and US-Israel BSF grant 2004353.

(C)2009 American Mathematical Society 
The earliest choice of biorthogonal polynomials $p_{n}$ related to numerical quadrature seems to be the Sidi polynomials $D_{n}^{(\alpha, \beta)}$ of [12, given by

$$
D_{n}^{(\alpha, \beta)}(x)=\sum_{i=0}^{n}(-1)^{n-i}\left(\begin{array}{c}
n \\
i
\end{array}\right)(\beta+i+1)^{\alpha+n} x^{i},
$$

where $\alpha, \beta>-1$. If $\alpha$ is a nonnegative integer, these admit the Rodrigues type representation

$$
D_{n}^{(\alpha, \beta)}(x)=(-1)^{n} x^{-\beta-1}\left(x \frac{d}{d x}\right)^{\alpha+n}\left[x^{\beta+1}(1-x)^{n}\right] .
$$

For general $\alpha, \beta>-1$, these polynomials satisfy the biorthogonality relation (1.3) with $w(x)=\left(\log x^{-1}\right)^{\alpha} x^{\beta},(a, b)=(0,1)$ and $\varphi_{j}(x)=(\log x)^{j-1}$; see [16]. That is,

$$
\int_{0}^{1} D_{n}^{(\alpha, \beta)}(x)\left(\log x^{-1}\right)^{j+\alpha} x^{\beta} d x=0, \quad 0 \leq j \leq n-1 .
$$

These polynomials first arose in the theory of convergence acceleration, but the associated quadrature rules turned out to be very accurate in numerical integration of functions with endpoint singularities. The asymptotic behavior of $D_{n}^{(0,0)}$ was determined in 4.

The biorthogonal polynomials of [13] and 17. satisfy (1.3) with $w(x)=x^{\alpha} e^{-x}$, $(a, b)=(0, \infty)$ and $\varphi_{j}(x)=e^{-\sigma_{j} x}$, for some appropriate $\left\{\sigma_{j}\right\}$; see [14] and [17]. A detailed study of the zero distribution of these polynomials was undertaken in [5]. Related biorthogonal polynomials have been investigated in [6] [7.

Now, the limited numerical computations carried out in [12] suggest that the weights $\left\{\lambda_{j}\right\}_{j=1}^{n}$ of the interpolatory rule generated by $D_{n}^{(0,0)}$ for the integral $\int_{0}^{1} f(x) d x$ might be positive for all $n$, but this was not known at the time of publication of [12]. In fact, this problem has remained open since 1980. It is that positivity that is one of the main results of this paper.

More generally, we shall consider a continuously differentiable, strictly increasing, function $\varphi:(a, b) \rightarrow \mathbb{R}$, and the monic polynomial $p_{n}$ of degree $n$ determined by the conditions

$$
\int_{a}^{b} p_{n} \varphi^{j} w=0, \quad 0 \leq j \leq n-1 .
$$

Thus we are choosing $\varphi_{j}=\varphi^{j-1}, 1 \leq j \leq n$, in (1.3).

Recall that a function $g$ is said to be $m$ absolutely monotone in an interval $J$ if $g^{(m)}$ exists there and

If

$$
g^{(j)}>0 \text { in } J \quad \text { for } 0 \leq j \leq m
$$

$$
(-1)^{j} g^{(j)}>0 \text { in } J \quad \text { for } 0 \leq j \leq m,
$$

$g$ is said to be $m$ completely monotone in $J$. Our main result is:

Theorem 1.1. Let $n \geq 1$ and $\varphi:(a, b) \rightarrow \mathbb{R}$ be a strictly increasing function with $n-1$ continuous derivatives, and let $\psi$ denote its inverse function, with domain of definition $I=\{\varphi(x): x \in(a, b)\}$. Assume that for each $\beta \in I$, the function

$$
g(t)=\frac{1}{\psi(\beta)-\psi(t)}, \quad t \in I \backslash\{\beta\},
$$


is $n-1$ absolutely monotone in $I \cap(-\infty, \beta)$ and $-g$ is $n-1$ completely monotone in $I \cap(\beta, \infty)$. Let $w:(a, b) \rightarrow(0, \infty)$ be such that $\int_{a}^{b} x^{j} \varphi(x)^{k} w(x) d x$ is defined and finite for $0 \leq j \leq n$ and $0 \leq k \leq n-1$. Let $p_{n}$ be the monic polynomial of degree $n$ determined by the biorthogonality conditions (1.5). Then the weights $\left\{\lambda_{j}\right\}_{j=1}^{n}$ in the interpolatory rule $I_{n}$ generated by $p_{n}$ and $w$ are all positive.

We can also prove positivity of the quadrature weights when the weight $w$ is replaced by $w|\varphi|$ :

Theorem 1.2. Assume the hypotheses of Theorem 1.1 and, in addition, that $\varphi$ is of one sign in $(a, b)$. Then the weights $\left\{\lambda_{j}\right\}_{j=1}^{n}$ in the interpolatory rule $I_{n}$ generated by $p_{n}$ and $\hat{w}=w|\varphi|$ are all positive.

Corollary 1.3. Let $\alpha, \beta>-1$ and $n \geq 1$. Let $w(x)=\left(\log x^{-1}\right)^{\alpha} x^{\beta}$ or $w(x)=$ $\left(\log x^{-1}\right)^{\alpha+1} x^{\beta}, x \in(0,1)$. Then the weights $\left\{\lambda_{j}\right\}_{j=1}^{n}$ in the interpolatory rule generated by the Sidi polynomials $D_{n}^{(\alpha, \beta)}$ and the weight $w$ are positive.

Remarks. (a) It is interesting that the conditions for the positivity of the weights $\left\{\lambda_{j}\right\}_{j=1}^{n}$, namely the absolute/complete monotonicity above, depend only on $\varphi$. Once these are satisfied, we obtain positivity for every weight $w$. Of course $p_{n}$, and hence the abscissas themselves, and the values of the weights, depend on $w$.

(b) The positivity of the weights has implications for convergence of the numerical quadratures as $n \rightarrow \infty$, as well as for associated rational approximations. It is curious that, despite the positivity of $\left\{\lambda_{j}\right\}_{j=1}^{n}$ for all $n$, it was shown in [4] that the zeros of the $\left\{D_{n}^{(0,0)}\right\}_{n=1}^{\infty}$ do not admit the arcsine distribution. All the abscissas of the classical quadratures admit the arcsine distribution, so this seems to be the first specific case of this phenomenon. The possible distributions of convergent interpolatory quadrature rules were explored in [1, where in particular it was shown that at least half of the nodes of such quadratures must have the arcsine distribution. (c) There are well-developed criteria [8, 9], 10, [1] for positivity of the weights $\left\{\lambda_{j}\right\}_{j=1}^{n}$ in general interpolatory quadratures, but these do not readily yield our results.

We can extend Theorem 1.2 to the case where the abscissas $\left\{x_{j}\right\}$ are generated by the polynomial $p_{n}$ satisfying (1.5), but the weights $\left\{\lambda_{j}\right\}$ are determined by a different weight $v$ :

Theorem 1.4. Let $n \geq 1$ and $\varphi:(a, b) \rightarrow \mathbb{R}$ be a strictly increasing function with $n-1$ continuous derivatives, and let $\psi$ denote its inverse function, with domain of definition $I=\{\varphi(x): x \in(a, b)\}$. Let $v, w:(a, b) \rightarrow(0, \infty)$ be such that $\int_{a}^{b} x^{j} \varphi(x)^{k} w(x) d x$ and $\int_{a}^{b} x^{j} \varphi(x)^{k} v(x) d x$ are defined and finite for $0 \leq j \leq n$ and $0 \leq k \leq n-1$. Let $p_{n}$ be the monic polynomial of degree $n$ determined by the biorthogonality conditions (1.5). Assume that for each $\beta \in I$, the function

$$
g(t)=\frac{(v / w)(\psi(t))}{\psi(\beta)-\psi(t)}, \quad t \in I \backslash\{\beta\},
$$

is $n-1$ absolutely monotone in $I \cap(-\infty, \beta)$ and $-g$ is $n-1$ completely monotone in $I \cap(\beta, \infty)$. Then the weights $\left\{\lambda_{j}\right\}_{j=1}^{n}$ in the interpolatory rule $I_{n}$ generated by $p_{n}$ and $v$ are all positive. 
Corollary 1.5. Let $\alpha, \beta>-1, \sigma \in(0,1)$ and $n \geq 1$. Let $w(x)=\left(\log x^{-1}\right)^{\alpha} x^{\beta}$ and $v(x)=\left(\log x^{-1}\right)^{\alpha} x^{\beta+\sigma}, x \in(0,1)$. Then the weights $\left\{\lambda_{j}\right\}_{j=1}^{n}$ in the interpolatory rule generated by the Sidi polynomials $D_{n}^{(\alpha, \beta)}$ and the weight $v$ are positive.

This paper is organized as follows: in Section 2, we shall present a more general criterion for positivity, and present examples. In Section 3, we shall prove Theorems 1.1, 1.2 and Corollary 1.3,

\section{A general CRIterion}

In this section, we assume that $\varphi_{j}:(a, b) \rightarrow \mathbb{R}, 1 \leq j \leq n$, and that $\left\{\varphi_{j}\right\}_{j=1}^{n}$ forms a Chebyshev system. That is, if

$$
\mathcal{S}=\left\{\sum_{j=1}^{n} c_{j} \varphi_{j}: c_{1}, c_{2}, \ldots, c_{n} \in \mathbb{R}\right\},
$$

then each element of $\mathcal{S}$ either has at most $n-1$ distinct zeros or is identically zero. Moreover, we assume that $w:(a, b) \rightarrow(0, \infty)$ is such that $\int_{a}^{b} x^{k} \varphi_{j}(x) w(x) d x$ is defined and finite for all $1 \leq j \leq n, 0 \leq k \leq n$. Moreover, $p_{n}$ is the monic polynomial of degree $n$ determined by the biorthogonality conditions (1.3). It is easily seen that $p_{n}$ exists and is given by

$$
p_{n}(x)=(-1)^{n} \frac{\operatorname{det}\left[\begin{array}{ccccc}
1 & x & x^{2} & \cdots & x^{n} \\
\int_{a}^{b} \varphi_{1} w & \int_{a}^{b} x \varphi_{1} w & \int_{a}^{b} x^{2} \varphi_{1} w & \cdots & \int_{a}^{b} x^{n} \varphi_{1} w \\
\int_{a}^{b} \varphi_{2} w & \int_{a}^{b} x \varphi_{2} w & \int_{a}^{b} x^{2} \varphi_{2} w & \cdots & \int_{a}^{b} x^{n} \varphi_{2} w \\
\int_{a}^{b} \varphi_{n} w & \int_{a}^{b} x \varphi_{n} w & \int_{a}^{b} x^{2} \varphi_{n} w & \cdots & \int_{a}^{b} x^{n} \varphi_{n} w
\end{array}\right]}{\operatorname{det}\left[\begin{array}{ccccc}
\int_{a}^{b} \varphi_{1} w & \int_{a}^{b} x \varphi_{1} w & \cdots & \int_{a}^{b} x^{n-1} \varphi_{1} w \\
\int_{a}^{b} \varphi_{2} w & \int_{a}^{b} x \varphi_{2} w & \cdots & \int_{a}^{b} x^{n-1} \varphi_{2} w \\
\int_{a}^{b} \varphi_{n} w & \int_{a}^{b} x \varphi_{n} w & \cdots & \int_{a}^{b} x^{n-1} \varphi_{n} w
\end{array}\right]} .
$$

That the denominator determinant is nonzero follows easily from our hypothesis that $\left\{\varphi_{j}\right\}_{j=1}^{n}$ forms a Chebyshev system. Likewise the latter hypothesis ensures that $p_{n}$ has $n$ real simple zeros in $(a, b)$.

Theorem 2.1. Assume that for each $\alpha \in(a, b)$, and each set of distinct points $\left\{s_{j}\right\}_{j=1}^{n-1} \subset(a, b) \backslash\{\alpha\}$, there exists $Q \in \mathcal{S}$ such that

$$
h(x)=\frac{1}{x-\alpha}-Q(x), \quad x \in(a, b) \backslash\{\alpha\}
$$

changes sign at each $s_{j}$ and has no other zeros in $(a, b)$. Then all the weights $\left\{\lambda_{j}\right\}_{j=1}^{n}$ in the interpolatory rule $I_{n}$ generated by $p_{n}$ and $w$ are all positive.

Proof. Let

$$
\ell_{j}(x)=\frac{p_{n}(x)}{p_{n}^{\prime}\left(x_{j}\right)\left(x-x_{j}\right)}
$$

denote the $j$ th fundamental polynomial of Lagrange interpolation, so that

$$
\lambda_{j}=\int_{a}^{b} \ell_{j} w
$$


Because of the biorthogonality condition (1.3), we can also write for any $Q \in \mathcal{S}$,

$$
\lambda_{j}=\int_{a}^{b} \frac{p_{n}(x)}{p_{n}^{\prime}\left(x_{j}\right)}\left\{\frac{1}{x-x_{j}}-Q(x)\right\} w(x) d x .
$$

We now choose $Q \in \mathcal{S}$ to be such that $\frac{1}{x-x_{j}}-Q(x)$ changes sign at each $x_{k}$ with $k \neq j$ and has no other zeros. (Thus $\alpha=x_{j}$ and $\left\{s_{k}\right\}_{k=1}^{n-1}=\left\{x_{k}\right\}_{k=1}^{n} \backslash\left\{x_{j}\right\}$.) Then the integrand

$$
f(x)=\frac{p_{n}(x)}{p_{n}^{\prime}\left(x_{j}\right)}\left\{\frac{1}{x-x_{j}}-Q(x)\right\}
$$

has "double" zeros at $x_{k}$ with $k \neq j$ and no other zeros. Here by a double zero, we mean that it has a zero at $x_{k}$, but does not change sign there. Moreover, there are no other zeros, and $f\left(x_{j}\right)=1$. It follows that $f>0$ in $(a, b)$ except at finitely many points, and so $\lambda_{j}>0$.

Examples. (I) Let $\varphi_{j}(x)=x^{j-1}, 1 \leq j \leq n$. Let $\alpha \in \mathbb{R}$ and $\left\{s_{j}\right\}_{j=1}^{n-1} \subset \mathbb{R} \backslash\{\alpha\}$ be distinct. Let $Q$ be the polynomial of degree $\leq n-2$ such that for $1 \leq j \leq n-1$,

$$
Q\left(s_{j}\right)=\frac{1}{s_{j}-\alpha} .
$$

Then

$$
S(x)=1-(x-\alpha) Q(x)
$$

is a polynomial of degree $\leq n-1$ that has zeros at $\left\{s_{j}\right\}_{j=1}^{n-1}$ and assumes the value 1 at $\alpha$. Hence it has simple zeros at $\left\{s_{j}\right\}_{j=1}^{n-1}$ and no other zeros. Then the hypotheses of Theorem 2.1 are satisfied, so we have another variant of a proof that the Gauss quadrature rule for the weight $w$ has positive weights.

(II) Let $\left\{I_{j}\right\}_{j=1}^{n}$ be intervals in $[0, \infty)$ with $I_{j}$ to the left of $I_{j+1}$ for each $j$. We allow their endpoints to touch. Let $\mu_{j}$ be a finite positive Borel measure on $I_{j}$ with positive measure on the interior of $I_{j}$. Let

$$
\varphi_{j}(x)=\int_{I_{j}} \frac{1}{x+t} d \mu_{j}(t), \quad x \in[0, \infty) .
$$

Let $\alpha$ and $\left\{s_{j}\right\}_{j=1}^{n-1}$ be distinct points in $(0, \infty)$. We assume that $\left\{s_{j}\right\}_{j=1}^{n}$ are in increasing order. Again, we choose $Q$ to be the linear combination of $\left\{\varphi_{j}\right\}_{j=1}^{n-1}$ that satisfies (2.2) for each $1 \leq j \leq n-1$. It is well known, and easy to see, that

$$
\frac{1}{x-\alpha}-Q(x)=\frac{\operatorname{det}\left[\begin{array}{ccccc}
\frac{1}{x-\alpha} & \varphi_{1}(x) & \varphi_{2}(x) & \cdots & \varphi_{n-1}(x) \\
\frac{1}{s_{1}-\alpha} & \varphi_{1}\left(s_{1}\right) & \varphi_{2}\left(s_{1}\right) & \cdots & \varphi_{n-1}\left(s_{1}\right) \\
\frac{1}{s_{2}-\alpha} & \varphi_{1}\left(s_{2}\right) & \varphi_{2}\left(s_{2}\right) & \cdots & \varphi_{n-1}\left(s_{2}\right) \\
\vdots & \vdots & \vdots & \ddots & \vdots \\
\frac{1}{s_{n-1}-\alpha} & \varphi_{1}\left(s_{n-1}\right) & \varphi_{2}\left(s_{n-1}\right) & \cdots & \varphi_{n-1}\left(s_{n-1}\right)
\end{array}\right]}{\operatorname{det}\left[\begin{array}{cccc}
\varphi_{1}\left(s_{1}\right) & \varphi_{2}\left(s_{1}\right) & \cdots & \varphi_{n-1}\left(s_{1}\right) \\
\varphi_{1}\left(s_{2}\right) & \varphi_{2}\left(s_{2}\right) & \cdots & \varphi_{n-1}\left(s_{2}\right) \\
\vdots & \vdots & \ddots & \vdots \\
\varphi_{1}\left(s_{n-1}\right) & \varphi_{2}\left(s_{n-1}\right) & \cdots & \varphi_{n-1}\left(s_{n-1}\right)
\end{array}\right]} .
$$


(Our discussion will show why the denominator determinant does not vanish.) Using the form of $\varphi_{j}$, we see that

$$
\frac{1}{x-\alpha}-Q(x)=\int_{I_{1}} \int_{I_{2}} \cdots \int_{I_{n-1}} \frac{D}{E} d \mu_{1}\left(t_{1}\right) d \mu_{2}\left(t_{2}\right) \cdots d \mu_{n-1}\left(t_{n-1}\right),
$$

where

$$
D=\operatorname{det}\left[\begin{array}{ccccc}
\frac{1}{x-\alpha} & \frac{1}{x+t_{1}} & \frac{1}{x+t_{2}} & \cdots & \frac{1}{x+t_{n-1}} \\
\frac{1}{s_{1}-\alpha} & \frac{1}{s_{1}+t_{1}} & \frac{1}{s_{1}+t_{2}} & \cdots & \frac{1}{s_{1}+t_{n-1}} \\
\frac{1}{s_{2}-\alpha} & \frac{1}{s_{2}+t_{1}} & \frac{1}{s_{2}+t_{2}} & \cdots & \frac{1}{s_{2}+t_{n-1}} \\
\vdots & \vdots & \vdots & \ddots & \vdots \\
\frac{1}{s_{n-1}-\alpha} & \frac{1}{s_{n-1}+t_{1}} & \frac{1}{s_{n-1}+t_{2}} & \cdots & \frac{1}{s_{n-1}+t_{n-1}}
\end{array}\right]
$$

and

$$
E=\operatorname{det}\left[\varphi_{k}\left(s_{j}\right)\right]_{1 \leq j, k \leq n-1} .
$$

Using the formula for Cauchy determinants [2, p. 195], we obtain

$$
D=\frac{\prod_{j=1}^{n-1}\left(s_{j}-x\right)\left(t_{j}+\alpha\right)}{(x-\alpha) \prod_{j=1}^{n-1}\left(x+t_{j}\right)\left(s_{j}-\alpha\right)} \frac{\prod_{1 \leq j<k \leq n-1}\left(s_{k}-s_{j}\right)\left(t_{k}-t_{j}\right)}{\prod_{1 \leq j, k \leq n-1}\left(t_{j}+s_{k}\right)} .
$$

We may assume that the $\left\{s_{j}\right\}$ are in increasing order. Moreover, our hypothesis that $I_{j}$ is to the left of $I_{j+1}$ forces $t_{k}-t_{j} \geq 0$ for $k \geq j$. Then we see that in the range of integration, the sign of $D$ is determined only by the sign of $\prod_{j=1}^{n-1}\left(s_{j}-x\right)$ and the sign of $(x-\alpha) \prod_{j=1}^{n-1}\left(s_{j}-\alpha\right)$. More precisely,

$$
D=\frac{\prod_{j=1}^{n-1}\left(s_{j}-x\right)}{(x-\alpha) \prod_{j=1}^{n-1}\left(s_{j}-\alpha\right)} C,
$$

where $C=C\left(s_{1}, s_{2}, \ldots, s_{n-1}, t_{1}, t_{2}, \ldots, t_{n-1}, \alpha, x\right)>0$ in the range of integration, except when $t_{k}=t_{j}$ for some $k>j$. Since each $\mu_{j}$ has positive measure on the interior of $I_{j}$, we see that $D$ changes sign, as a function of $x$, only at $\left\{s_{j}\right\}_{j=1}^{n-1}$, and has no other zeros. Similarly $E>0$. Thus the hypotheses of Theorem 2.1 are fulfilled.

Two special cases are of interest: when $\mu_{j}$ is a unit mass at $\beta_{j} \in I_{j}^{0}$, for each $j$, so that

$$
\varphi_{j}(x)=\frac{1}{x+\beta_{j}}, \quad 1 \leq j \leq n .
$$

This case can also be reduced to that of a Gauss quadrature for the weight $w(x) /$ $\prod_{j=1}^{n}\left(x+\beta_{j}\right)$. A second is where $I_{j}=\left[a_{j}, b_{j}\right]$ and $d \mu_{j}(t)=d t$ on $I_{j}$, so that

$$
\varphi_{j}(x)=\log \frac{x+b_{j}}{x+a_{j}}, \quad 1 \leq j \leq n .
$$




\section{Proofs}

We shall make use of

Lemma 3.1. Let $-\infty \leq \alpha<\xi<\infty$, let $m \geq 1$, and $f:(\alpha, \xi] \rightarrow \mathbb{R}$ be a function for which $f^{(m+1)}$ exists in $(\alpha, \xi]$, and $f^{(j)}>0$ in $(\alpha, \xi], 0 \leq j \leq m+1$. Let $\pi_{m}$ be a polynomial of degree $\leq m$, and let $m_{1}$ be the total multiplicity of zeros of $f-\pi_{m}$ in $[\alpha, \xi]$, and $m_{2}$ be the total multiplicity of zeros of $\pi_{m}$ in $[\xi, \infty)$. Then

$$
m_{1}+m_{2} \leq m+1
$$

Proof. For the case where $\alpha=-\infty$, this appears in [3, p. 30, Lemma 5.3]. The proof for the above case is exactly the same. Alternatively, one can extend $f$ to $(-\infty, \alpha]$ in such a way that the first $m+1$ derivatives exist and remain positive, and apply Freud's lemma as stated. The number of zeros of $f-\pi_{m}$ in $[\alpha, \xi]$ does not exceed the number in $(-\infty, \xi]$.

Lemma 3.2. Let $\psi$ and $I$ be as in Theorem 1.1. Let $\beta \in I$. Let $\left\{y_{j}\right\}_{j=1}^{n-1}$ be distinct points in $I \backslash\{\beta\}$ and

$$
g(t)=\frac{1}{\psi(\beta)-\psi(t)}, \quad t \in I \backslash\{\beta\} .
$$

Then there exists a polynomial $R$ of degree $\leq n-2$ such that $g-R$ has sign changes at $\left\{y_{j}\right\}_{j=1}^{n-1}$ and no other zeros in $I$.

Proof. Define

$$
f_{1}(t)= \begin{cases}\frac{1}{\psi(\beta)-\psi(t)}, & t \in I \cap(-\infty, \beta), \\ 0, & t \in I \cap(\beta, \infty)\end{cases}
$$

and

$$
f_{2}(t)= \begin{cases}0, & t \in I \cap(-\infty, \beta), \\ \frac{1}{\psi(\beta)-\psi(t)}, & t \in I \cap(\beta, \infty) .\end{cases}
$$

Then

$$
g=f_{1}+f_{2} .
$$

The idea is to apply Lemma 3.1 to $f_{1}$ giving a suitable polynomial $S$, and apply Lemma 3.1 to $-f_{2}(-t)$, on a suitable interval, giving a polynomial $T$, after mapping the variable back. We then set

$$
R=S+T \text {. }
$$

Let us assume

$$
y_{1}<y_{2}<\cdots<y_{j-1}<\beta<y_{j}<\cdots<y_{n-1},
$$

and set $y_{0}=\inf I$ and $y_{n}=\sup I$. Let $S$ be the polynomial of degree $\leq n-2$ that interpolates to $f_{1}$ at $\left\{y_{j}\right\}_{j=1}^{n-1}$. Then $f_{1}-S$ has $m_{1} \geq j-1$ zeros in $I \cap(-\infty, \beta)$ and $S$ has $m_{2} \geq n-j$ zeros in $(\beta, \infty)$. By Lemma 3.1 .

$$
\begin{aligned}
m_{1}+m_{2} & \leq n-1 \\
& \Rightarrow m_{1}=j-1 \text { and } m_{2}=n-j .
\end{aligned}
$$

It follows that $f_{1}-S$ has simple zeros at $y_{1}, y_{2}, \ldots, y_{j-1}$, and $S$ has simple zeros at $y_{j}, y_{j+1}, \ldots, y_{n-1}$, and no other zeros. From this we can determine the sign pattern of $f_{1}-S$ and $S$. See Figure 1 . 


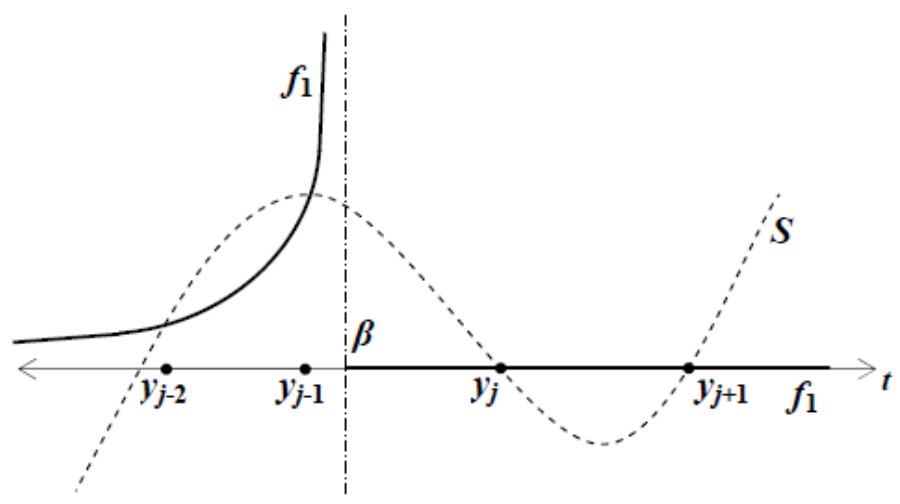

Figure 1

By choosing $\xi=y_{j-1}$ in Lemma 3.1, we see that $S$ has no zeros in $\left(y_{j-1}, y_{j}\right)$. Moreover, $S\left(y_{j-1}\right)=f_{1}\left(y_{j-1}\right)>0=S\left(y_{j}\right)$. Thus, as $f_{1}-S$ has a sign change at $y_{j-1}$, as does $S$ at $y_{j}$ :

$$
\begin{array}{r}
S>0 \text { in }\left(y_{j-1}, y_{j}\right) ; \\
f_{1}-S>0 \text { in }\left(y_{j-1}, \beta\right) .
\end{array}
$$

Because of the simplicity of the zeros of $S$ and $f_{1}-S$ at the $\left\{y_{k}\right\}$, we deduce that

$$
\begin{aligned}
\operatorname{sign}(S) & =(-1)^{k} \text { in }\left(y_{j-1+k}, y_{j+k}\right), & & 0 \leq k \leq n-j ; \\
\operatorname{sign}\left(f_{1}-S\right) & =(-1)^{k} \text { in }\left(y_{j-k-1}, y_{j-k}\right), & & 1 \leq k \leq j-1 .
\end{aligned}
$$

Next, let $T$ be the polynomial of degree $\leq n-2$ that interpolates to $f_{2}$ at $\left\{y_{j}\right\}_{j=1}^{n-1}$. Since $-f_{2}(-t)$ and $-T(-t)$ satisfy the hypotheses of Lemma 3.1 on an appropriate interval, we see that by exactly the same reasoning as for $f_{2}$, that $T$ has simple zeros at $y_{1}, y_{2}, \ldots, y_{j-1}$, and $f_{2}-T$ has simple zeros at $y_{j}, y_{j+1}, \ldots, y_{n-1}$, and no other zeros. Moreover, $T\left(y_{j-1}\right)=0>f_{2}\left(y_{j}\right)=T\left(y_{j}\right)$. See Figure 2 .

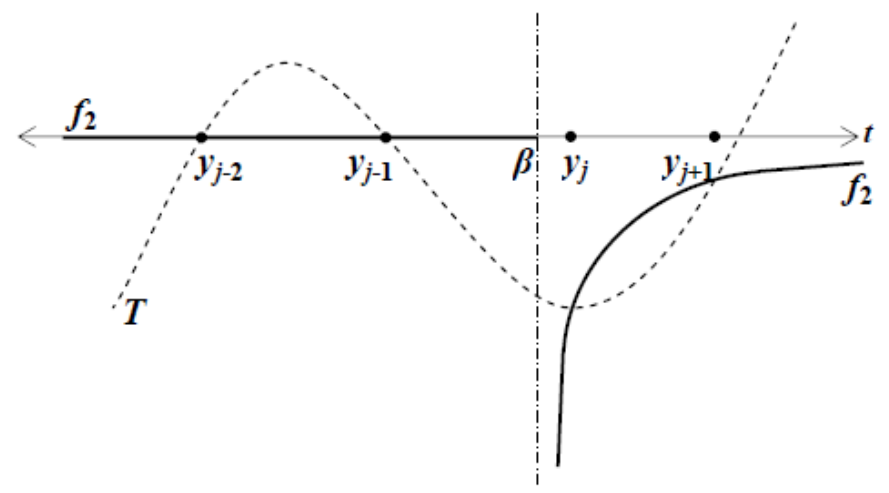

Figure 2 
As $f_{2}-T$ has a sign change at $y_{j}$, as does $T$ at $y_{j-1}$,

$$
\begin{aligned}
T & <0 \text { in }\left(y_{j-1}, y_{j}\right) ; \\
f_{2}-T & <0 \text { in }\left(\beta, y_{j}\right) .
\end{aligned}
$$

Because of the simplicity of the zeros of $T$ and $f_{2}-T$ at the $\left\{y_{k}\right\}$, we deduce that

$$
\begin{aligned}
\operatorname{sign}(T) & =(-1)^{k+1} \text { in }\left(y_{j-k-1}, y_{j-k}\right), & & 1 \leq k \leq j-1 ; \\
\operatorname{sign}\left(f_{2}-T\right) & =(-1)^{k+1} \text { in }\left(y_{j-1+k}, y_{j+k}\right), & & 1 \leq k \leq n-j .
\end{aligned}
$$

From (3.4) and (3.6), we deduce that in $\left(y_{j-k-1}, y_{j-k}\right), 1 \leq k \leq j-1$, both $f_{1}-S$ and $-T$ have sign $(-1)^{k}$, so

$$
g-R=\left(f_{1}-S\right)-T \text { has sign }(-1)^{k} .
$$

Next, from (3.3) and (3.7), we deduce that in $\left(y_{j-1+k}, y_{j+k}\right), 1 \leq k \leq n-j$, both $f_{2}-T$ and $-S$ have sign $(-1)^{k+1}$, so

$$
g-R=\left(f_{2}-T\right)-S \text { has sign }(-1)^{k+1} .
$$

Finally in $\left(y_{j-1}, \beta\right), f_{1}-S$ and $-T$ are positive, so $g-R$ is positive; and in $\left(\beta, y_{j}\right)$, $f_{2}-T$ and $-S$ are negative, so $g-R$ is negative. We have shown that $g-R$ has sign changes at all the $\left\{y_{j}\right\}_{j=1}^{n-1}$ and no other zeros.

Proof of Theorem 1.1. We must verify that for each $\alpha \in(a, b)$, and each set of distinct $\left\{s_{j}\right\}_{j=1}^{n-1}$ in $(a, b) \backslash\{\alpha\}$, there is a generalized polynomial

$$
Q(x)=\sum_{j=0}^{n-1} c_{j} \varphi(x)^{j}
$$

such that $\frac{1}{x-\alpha}-Q(x)$ changes sign at each of the $\left\{s_{j}\right\}_{j=1}^{n-1}$ and has no other zeros in $(a, b)$. We make the changes of variables $x=\psi(t), \alpha=\psi(\beta), s_{j}=\psi\left(y_{j}\right)$. Also set

$$
R(t)=\sum_{j=0}^{n-1} c_{j} t^{j}
$$

Then we have to find a polynomial $R$ of degree $\leq n-1$ such that $\frac{1}{\psi(t)-\psi(\beta)}-R(t)$ has sign changes at $\left\{y_{j}\right\}_{j=1}^{n-1}$ and no other zeros in $I$. Such a polynomial, even of degree $\leq n-2$, was constructed in the previous lemma (just multiply the polynomial there by -1 ). Then Theorem 2.1 gives the positivity of the weights generated by $p_{n}$ and $w$.

Proof of Theorem 1.2, We return to the idea of the proof of Theorem 2.1, Let $\sigma$ denote the sign of $\varphi$ in $(a, b)$. The $j$ th weight in the quadrature generated by $p_{n}$ and $\hat{w}=w|\varphi|$ is

$$
\begin{aligned}
\lambda_{j} & =\int_{a}^{b} \frac{p_{n}(x)}{p_{n}^{\prime}\left(x_{j}\right)} \frac{1}{x-x_{j}} w(x)|\varphi(x)| d x \\
& =\sigma \int_{a}^{b} \frac{p_{n}(x)}{p_{n}^{\prime}\left(x_{j}\right)}\left\{\frac{1}{x-x_{j}}-R(\varphi(x))\right\} w(x) \varphi(x) d x,
\end{aligned}
$$

for any algebraic polynomial $R$ of degree $\leq n-2$. Of course, we have used the biorthogonality condition (1.5). The trick of the proof is to recall that in the proof of Theorem 1.2, we only needed a polynomial $R$ of degree $n-2$ (and not $n-1$ ) 
to ensure that $\frac{1}{x-x_{j}}-R(\varphi(x))$ has sign changes at $\left\{x_{k}\right\}_{k=1}^{n} \backslash\left\{x_{j}\right\}$, and no other zeros in $(a, b)$. The result then follows.

Proof of Corollary 1.3 for $w(x)=\left(\log x^{-1}\right)^{\alpha} x^{\beta}$. The polynomials $D_{n}^{(\alpha, \beta)}$ are biorthogonal to $(\log x)^{j}, 0 \leq j \leq n-1$, with the weight $w(x)=\left(\log x^{-1}\right)^{\alpha} x^{\beta}$, $x \in(0,1)$. Here $\varphi(x)=\log x$ and the inverse function is $\psi(t)=e^{t}, t \in(-\infty, 0)$. We have to show that for each $\gamma \in(-\infty, 0)$, the function

$$
g(t)=\frac{1}{e^{\gamma}-e^{t}}, \quad t \in(-\infty, 0) \backslash\{\gamma\}
$$

is $n-1$ absolutely monotone in $(-\infty, \gamma)$ and $-g$ is $n-1$ completely monotone in $(\gamma, 0)$. For $t \in(-\infty, \gamma)$, we write

$$
g(t)=\frac{1}{e^{\gamma}\left(1-e^{t-\gamma}\right)}=e^{-\gamma} \sum_{k=0}^{\infty}\left(e^{t-\gamma}\right)^{k} .
$$

Then for such a $t$, and each fixed $j \geq 0$,

$$
g^{(j)}(t)=e^{-\gamma} \sum_{k=0}^{\infty} k^{j}\left(e^{t-\gamma}\right)^{k}>0,
$$

and the term-by-term differentiation is justified by (locally) uniform convergence of the differentiated series. For $t \in(\gamma, \infty)$, we have

$$
-g(t)=\frac{1}{e^{t}\left(1-e^{\gamma-t}\right)}=e^{-\gamma} \sum_{k=0}^{\infty}\left(e^{\gamma-t}\right)^{k+1}
$$

and then

$$
-(-1)^{j} g^{(j)}(t)=e^{-\gamma} \sum_{k=0}^{\infty}(k+1)^{j}\left(e^{\gamma-t}\right)^{k+1}>0 .
$$

Thus the hypotheses of Theorem 1.1 are fulfilled.

Proof of Corollary 1.3 for $w(x)=\left(\log x^{-1}\right)^{\alpha+1} x^{\beta}$. This follows immediately from Theorem 1.2 with $|\varphi(x)|=|\log x|=\log x^{-1}$, and the previous considerations.

Proof of Theorem 1.4. The weights in the quadrature rule generated by $p_{n}$ and $v$ satisfy

$$
\lambda_{j}=\int_{a}^{b} \frac{p_{n}(x)}{p_{n}^{\prime}\left(x_{j}\right)}\left[\frac{(v / w)(x)}{x-x_{j}}-Q(\varphi(x))\right] w(x) d x,
$$

for any algebraic polynomial $Q$ of degree $\leq n-1$, in view of (1.5). We can then follow the same proof as is in Theorems 2.2 and 1.2, with an obvious modification of Lemma 3.2.

Proof of Corollary 1.5. Following the same lines as in the proof of Corollary 1.3 . we let

$$
g(t)=\frac{(v / w)(\psi(t))}{\psi(\gamma)-\psi(t)}=\frac{e^{\sigma t}}{e^{\gamma}-e^{t}}, \quad t \in(-\infty, 0) \backslash\{\gamma\} .
$$

For $t \in(-\infty, \gamma)$, we see that

$$
g(t)=\sum_{k=0}^{\infty} e^{(k+\sigma) t-(k+1) \gamma},
$$


and for $t \in(\gamma, 0)$,

$$
-g(t)=\sum_{k=0}^{\infty} e^{(\sigma-k-1) t+k \gamma},
$$

so can proceed as for Corollary 1.3 .

\section{REFERENCES}

[1] T. Bloom, D. S. Lubinsky, and H. Stahl, What Distributions of Points are Possible for Convergent Sequences of Interpolatory Integration Rules?, Constr. Approx., 9(1993), 41-58. MR.1198522 (94f:41033)

[2] E. W. Cheney, Introduction to Approximation Theory, Chelsea, New York, 1982. MR.1656150 (99f:41001)

[3] G. Freud, Orthogonal Polynomials, Akademiai Kiado, Budapest, 1971.

[4] D.S. Lubinsky and A. Sidi, Strong Asymptotics for Polynomials Biorthogonal to Powers of $\log x$, Analysis, 14(1994), 341-379. MR1310619 (96b:42031)

[5] D.S. Lubinsky and A. Sidi, Zero Distribution of Composite Polynomials and Polynomials Biorthogonal to Exponentials, Constructive Approximation, 28(2008), 343-371. MR2453371

[6] D. S. Lubinsky and I. Soran, Weights whose Biorthogonal Polynomials admit a Rodrigues Formula, JMAA, 324(2006), 805-819. MR2265082(2008b:33022)

[7] D. S. Lubinsky and H. Stahl, Some Explicit Biorthogonal Polynomials, (in) Approximation Theory XI (eds. C. K. Chui, M. Neamtu, L. L. Schumaker), Nashboro Press, Brentwood, TN, 2005, pp. 279-285. MR2126686 (2005k:42073)

[8] F. Peherstorfer, Characterization of Positive Quadrature Formulas, SIAM J. Math. Anal., 12(1981), 935-942. MR635246 (82m:65021)

[9] F. Peherstorfer, Characterization of Quadrature Formulas II, SIAM J. Math. Anal., 15(1984), 1021-1030. MR755862 (86a:65025)

[10] F. Peherstorfer, Positive Quadrature Formulas III: Asymptotics of Weights, Math. Comp., 77(2008), 2241-2259. MR2429883

[11] H. J. Schmid, A Note on Positive Quadrature Rules, Rocky Mountain J. Math., 19(1989), 395-404. MR1016190 (90k:41041)

[12] A. Sidi, Numerical Quadrature and Non-Linear Sequence Transformations: Unified Rules for Efficient Computation of Integrals with Algebraic and Logarithmic Endpoint Singularities, Math. Comp., 35(1980), 851-874. MR:572861 (81h:65017)

[13] A. Sidi, Numerical Quadrature Rules for Infinite Range Integrals, Math. Comp., 38(1982), 127-142. MR637291 (82m:65022)

[14] A. Sidi, Problems 5-8, (in) Numerical Integration III (eds. H. Brass and G. Hämmerlin), Birkhäuser, Berlin, 1988, pp. 321-325.

[15] A. Sidi, Practical Extrapolation Methods: Theory and Applications, Cambridge University Press, Cambridge, 2003. MR 1994507 (2004e:65005)

[16] A. Sidi and D. S. Lubinsky, On the Zeros of Some Polynomials that Arise in Numerical Quadrature and Convergence Acceleration, SIAM J. Numer. Anal., 20(1983), 400-405. MR694528 (84f:65046)

[17] A. Sidi and D. S. Lubinsky, Biorthogonal Polynomials and Numerical Integration Formulas for Infinite Intervals, Journal of Numerical Analysis, Industrial and Applied Mathematics, 2(2007), 209-226. MR2376090(2008m:33029)

School of Mathematics, Georgia Institute of Technology, Atlanta, Georgia 30332 0160

E-mail address: lubinsky@math.gatech.edu

Department of Computer Science, Technion-Israel Institute of Technology, Haifa 32000 ISRAEL

E-mail address: asidi@cs.technion.ac.il 